Research Paper

\title{
Prognostic Nutritional Index identifies risk of early progression and survival outcomes in Advanced Non-small Cell Lung Cancer patients treated with PD-1 inhibitors
}

\author{
Na Liu*, Aimin Jiang*, Xiaoqiang Zheng, Xiao Fu, Haoran Zheng, Huan Gao, Jingjing Wang, Xuan Liang, Tao \\ Tian, Zhiping Ruan ${ }^{凶}, \mathrm{Yu} \mathrm{Yao}^{\bowtie}$ \\ Department of Medical Oncology, The First Affiliated Hospital of Xi'an Jiaotong University, No.277 Yanta West Road, Xi'an, Shaanxi 710061, People's Republic \\ of China. \\ *These authors contributed equally to this work. \\ $\triangle$ Corresponding authors: Zhiping Ruan, Department of Medical Oncology, The First Affiliated Hospital of Xi'an Jiaotong University, No. 277 Yanta West \\ Road, Xi'an, Shaanxi 710061, People's Republic of China. Tel: +86-29-85324086; Fax: +86-29-85324086; E-mail: zopor@163.com; Yu Yao, Department of Medical \\ Oncology, The First Affiliated Hospital of Xi'an Jiaotong University, No. 277 Yanta West Road, Xi'an, Shaanxi 710061, People's Republic of China. Tel: \\ +86-29-85324086; Fax: +86-29-85324086; E-mail: 13572101611@163.com. \\ (C) The author(s). This is an open access article distributed under the terms of the Creative Commons Attribution License (https://creativecommons.org/licenses/by/4.0/).
} See http://ivyspring.com/terms for full terms and conditions.

Received: 2020.11.15; Accepted: 2021.02.25; Published: 2021.03.15

\begin{abstract}
Background: The prognostic nutritional index (PNI) is related to the prognosis of multiple malignancies. This study investigated whether the PNI has prognostic value in advanced non-small cell lung cancer (NSCLC) patients treated with programmed death 1 (PD-1) inhibitors.

Methods: We retrospectively analyzed advanced NSCLC patients treated with PD-1 inhibitors from July 2018 to December 2019. Pretreatment PNI was calculated by peripheral lymphocyte count and serum albumin level, and the cut-off value was determined. Subsequently, we investigated the relationship between PNI and early progression, and evaluated its prognostic role on survival outcomes. Ultimately, based on the results of survival analysis, a nomogram was established.

Results: A total of 123 patients were included. Of these, 24 (19.5\%) patients had experienced early progression. Multivariate logistic analysis indicated that low PNI (odds ratio, 3.709, 95\% confidence interval $[\mathrm{Cl}], 1.354-10.161 ; P=0.011)$ was closely correlated with early progression. Moreover, multivariate Cox regression analysis confirmed that low PNI was an independent risk factor for progression-free survival (hazard ratio [HR], 2.698, 95\% Cl, 1.752-4.153; $P<0.001$ ) and overall survival (HR, 7.222, 95\% Cl, 4.081-12.781; $P<0.001$ ), respectively. The prediction accuracy of nomogram based on $\mathrm{PNI}$ is moderate.

Conclusion: PNI was an independent predictor of early progression and survival outcomes in advanced NSCLC patients treated with PD-1 inhibitors.
\end{abstract}

Key words: non-small cell lung cancer; prognosis nutritional index; PD-1 inhibitors; prognosis; nomogram

\section{Introduction}

According to cancer statistics, 2020, lung cancer (LC) is the second incidence rate and the first death rate cancer among men and women, accounting for $13 \%$ of all cancer diagnoses and $23 \%$ of all cancer-related deaths [1]. Non-small cell lung cancer (NSCLC) accounts for more than $85 \%$ of LC, and the 5 -year overall survival (OS) rate in advanced stage patients is less than 5\% [2]. In recent years, with the improvement of biological understanding of LC and the remarkable progress of immunotherapy in LC, the OS of advanced NSCLC patients has been prominently improved.

Immune checkpoint inhibitors (ICIs) have been applied in the clinical practice. Early-stage clinical trials have shown that approximately $14 \%-20 \%$ of advanced NSCLC patients receiving ICIs had a rapid and sustained response. The results of KEYNOTE-024 and KEYNOTE-042 trials suggested that 
pembrolizumab monotherapy was approved for the first-line treatment in patients with programmed death ligand-1 (PD-L1) expression $\geq 50 \%$ [3, 4]. However, according to the results of KEYNOTE-189 and KEYNOTE-407, patients can benefit from pembrolizumab plus chemotherapy regardless of their PD-L1 expression status [5, 6, 7]. In the CheckMate-227 trial, patients with high tumor mutation burden (TMB) could benefit from nivolumab combined with ipilimumab, and TMB may be an ideal predictor in these individuals. However, this study also showed that the clinical benefit of patients was not related to PD-L1 expression status. Analysis of blood TMB (bTMB) in POPLAR and OAK trials indicated that patients with high $\mathrm{bTMB}$ could benefit more from atezolizumab $[8,9]$. Due to the lack of correlation between TMB and PD-L1 expression $[10,11]$, the combination may not exert a synergistic effect. Currently, immunotherapy still lacks effective biomarkers. PD-L1, TMB, tumor-infiltrating lymphocytes (TILs), and mismatch repair (MMR) cannot be used as golden indicators for choosing the benefit population.

Recently, mounting studies have highlighted the significance of nutritional and immune status in oncological patients, suggesting that it is pivotal in cancer progression and prognosis $[12,13]$. Prognostic nutritional index (PNI) is obtained by the level of serum albumin and peripheral lymphocytes, which can reflect the nutritional and immune status of patients [14]. Several studies have confirmed that PNI is related to the response and prognosis of patients with chemotherapy or chemoradiotherapy [15, 16]. Moreover, the indicator is readily available, non-invasion and dynamically monitored, with a high clinical application prospect. However, whether PNI is related to the clinical outcomes of advanced NSCLC patients treated with programmed death-1 (PD-1) inhibitors remains unclear. Therefore, our study evaluated the impact of PNI on early progression and prognosis in those patients.

\section{Methods}

\section{Patient selection}

We collected the clinical information of advanced NSCLC patients who received PD-1 inhibitors in the First Affiliated Hospital of Xi'an Jiaotong University from July 2018 to December 2019. The inclusion criteria were as follows: (1) age over 18 years old; (2) patients who were diagnosed by histopathology or cytopathology as NSCLC; (3) stage IIIB-IV treated with PD-1 inhibitors; (4) Eastern Cooperative Oncology Group Performance Status (ECOG PS) score at 0-2; (5) all patients had available hematological parameters (peripheral lymphocyte count and serum albumin level) and evaluable imaging data before treatment; (6) at least one cycle of PD-1 inhibitors treatment (monotherapy or combination therapy). The exclusion criteria were: (1) patients with incomplete clinicopathological data and follow-up information; (2) combined with autoimmune diseases, hematologic disorders, and other diseases; (3) a history of using steroid within two weeks; (4) infectious diseases before PD-1 inhibitors treatment; (5) patients with other primary carcinomas. The study was approved by the Ethics Committee of our hospital (XJTU1AF2020LSK-141).

Basic clinicopathological data were collected from patients who correspond to the standard. Clinical responses were assessed according to iRECIST criteria. Early progression was defined as progression or cancer-related death occurring within 8 weeks after receiving PD-1 inhibitors. Progressionfree survival (PFS) was calculated from the start of treatment with PD-1 inhibitors to disease progression or death. And OS was calculated from the start to death for any cause. The last follow-up was performed on August 30, 2020.

\section{Prognostic nutritional index}

The calculation formula of PNI was: serum albumin $(\mathrm{g} / \mathrm{L})+5 \times$ peripheral lymphocyte count $\left(10^{9} / \mathrm{L}\right)$, and the results within one week before treatment were required. The receiver operating characteristic (ROC) curve was established, and the state variable value corresponding to the maximum value of Youden index was the PNI cut-off value. According to the cut-off value, patients were divided into L- and H- PNI groups.

\section{Statistical analysis}

The Chi-square or Fisher's exact test was used to compare clinicopathological data between H-PNI and L-PNI groups. Continuous variables are compared using student's t-test. The relationship between clinical variables and the risk of early progression was assessed by logistic regression analysis. The survival curves of H-PNI and L-PNI groups were drawn by Kaplan-Meier (K-M) method. The Cox regression analysis was utilized to determine the prognostic factors of PFS and OS. Finally, a nomogram related to prognosis was developed, and the predictive accuracy of the model was assessed. All statistical analyses were two-sided probability tests $(\alpha=0.05), P<0.05$ was considered the difference to be statistically significant. The above statistical analysis was performed using IBM SPSS Statistic 18.0 and R Studio 3.6.0. 


\section{Results}

\section{Patient characteristics}

A total of 123 patients were enrolled in this study (Table 1). The average age at the time of diagnosis was $59.9 \pm 11.3$ years. Male patients account for the majority $(79.7 \%) .60 .2 \%$ of patients were lung adenocarcinoma (LUAD), 37.4\% were lung squamous cell carcinoma (LUSC), and $2.4 \%$ were other types (2 cases of adenosquamous carcinoma, 1 case of large cell carcinoma). Stage IIIB/IIIC accounted for $18.7 \%$. All of them had used at least one cycle of PD-1 inhibitors during the study period $(17.9 \%$ for nivolumab, $42.3 \%$ for pembrolizumab, $25.2 \%$ for sintilimab, $8.1 \%$ for camrelizumab, and $6.5 \%$ for toripalimab), of which $42.3 \%$ were first-line therapy and $78 \%$ were combined chemotherapy. $27.6 \%$ of patients had known the PD-L1 expression status (1-49\%, 12 cases; $\geq 50 \%, 12$ cases; and negative, 10 cases). Patients with driver genomic alterations account for $17.1 \%$ (epidermal growth factor receptor [EGFR], 16.3\%; ROS1 proto-oncogene receptor tyrosine kinase [ROS1], $0.8 \%$ ). $13.0 \%$ of patients had liver metastases, $16.3 \%$ had central nervous system (CNS) metastases, and $30.9 \%$ had bone metastases. During the treatment, $24.4 \%$ of patients developed immune-related adverse events (irAEs).

ROC curve showed that the optimal PNI cut-off value was 46.05 (Supplementary Figure S1). The corresponding sensitivity and specificity were 0.833 and 0.683 , respectively (area under the ROC curve was 0.780). According to the cut-off value (46.05), patients were divided into L-PNI (53 cases) and H-PNI (70 cases) groups. As shown in Table 1, the relationship between PNI and clinical characteristics of patients was summarized. The two groups had significant differences in CNS metastasis $(P=0.031)$ and early progression $(P=0.009)$.

\section{Early progression}

Twenty-four patients (19.5\%) had developed early progression (within 8 weeks). Figure 1 was a box plot of the relationship between early progression and PNI. Compared with non-early progression patients, the PNI level of patients with early progression was remarkably decreased $(P=0.001)$. Furthermore, logistic regression analysis showed that the therapy line, driver genomic alterations, and PNI were associated with early progression. The multivariate analysis confirmed that the therapy line (odds ratio [OR], 5.860, 95\% confidence interval [CI], 1.544-22.246; $P=0.009)$ and PNI (OR, 3.709, 95\% CI, 1.354-10.161; $P$ $=0.011$ ) were independent predictors of early progression.

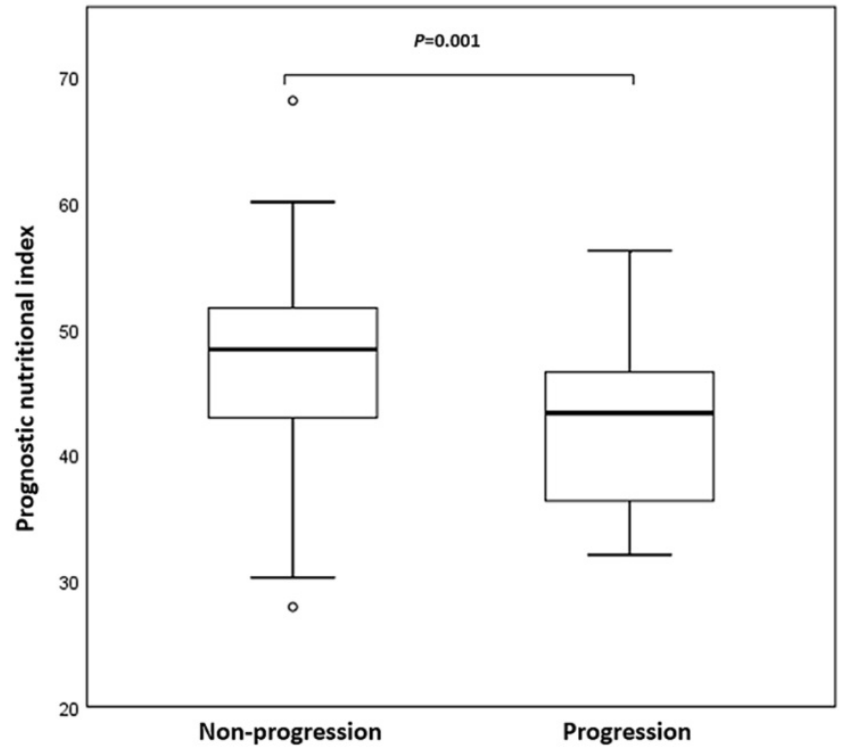

Figure 1. Box plot of prognostic nutritional index (PNI) in non-small cell lung cancer (NSCLC) patients with non-progression and early progression. Early progression was defined as progression within 8 weeks after initiation of programmed death 1 (PD-1) inhibitors.

Table 1. Baseline clinical characteristics of NSCLC patients treated with PD-1 inhibitors

\begin{tabular}{|c|c|c|c|c|}
\hline Clinical characteristics & Overall [n (\%)] & PNI $>46.05[\mathrm{n}(\%)]$ & $\mathrm{PNI} \leq 46.05[\mathrm{n}(\%)]$ & $\begin{array}{l}P \\
\text { value }\end{array}$ \\
\hline Total & 123 & $70(56.9)$ & $53(43.1)$ & \\
\hline Age & & & & 0.898 \\
\hline$\leq 65$ & $82(66.7)$ & $47(67.1)$ & $35(66.0)$ & \\
\hline$>65$ & $41(33.3)$ & $23(32.9)$ & $18(34.0)$ & \\
\hline Gender & & & & 0.088 \\
\hline Male & $98(79.7)$ & $52(74.3)$ & $46(86.8)$ & \\
\hline Female & $25(20.3)$ & $18(25.7)$ & 7 (13.2) & \\
\hline Smoking status & & & & 0.946 \\
\hline Never & $46(37.4)$ & $26(37.1)$ & $20(37.7)$ & \\
\hline Current/previous & $77(62.6)$ & $44(62.9)$ & $33(62.3)$ & \\
\hline ECOG PS & & & & 0.101 \\
\hline 0 & $84(68.3)$ & $52(74.3)$ & $32(60.4)$ & \\
\hline $1-2$ & $39(31.7)$ & $18(25.7)$ & $21(39.6)$ & \\
\hline History & & & & 0.877 \\
\hline LUAD & $74(60.2)$ & $43(61.4)$ & $31(58.5)$ & \\
\hline LUSC & $46(37.4)$ & $25(35.7)$ & $21(39.6)$ & \\
\hline Other & $3(2.4)$ & $2(2.9)$ & $1(1.9)$ & \\
\hline Stage & & & & 0.611 \\
\hline IIIB/IIIC & $23(18.7)$ & $12(17.1)$ & $11(20.8)$ & \\
\hline IV & $100(81.3)$ & $58(82.9)$ & $42(79.2)$ & \\
\hline Therapy line & & & & 0.604 \\
\hline 1 & $52(42.3)$ & $31(44.3)$ & $21(39.6)$ & \\
\hline$\geq 2$ & $71(57.7)$ & $39(55.7)$ & $32(60.4)$ & \\
\hline Immunotherapy drug & & & & 0.753 \\
\hline Nivolumab & $22(17.9)$ & $10(14.3)$ & $12(22.6)$ & \\
\hline Pembrolizumab & $52(42.3)$ & $30(42.9)$ & $22(41.5)$ & \\
\hline Sintilimab & $31(25.2)$ & $18(25.7)$ & $13(24.5)$ & \\
\hline Camrelizumab & $10(8.1)$ & $7(10.0)$ & $3(5.7)$ & \\
\hline Toripalimab & $8(6.5)$ & $5(7.1)$ & $3(5.7)$ & \\
\hline Regimen & & & & 0.055 \\
\hline Monotherapy & $27(22.0)$ & $11(15.7)$ & $16(30.2)$ & \\
\hline Combination therapy & $96(78.0)$ & $59(84.3)$ & $37(69.8)$ & \\
\hline Liver metastasis & & & & 0.254 \\
\hline No & $107(87.0)$ & $63(90.0)$ & $44(83.0)$ & \\
\hline Yes & $16(13.0)$ & $7(10.0)$ & $9(17.0)$ & \\
\hline CNS metastasis & & & & 0.031 \\
\hline No & $103(83.7)$ & $63(90.0)$ & $40(75.5)$ & \\
\hline Yes & $20(16.3)$ & $7(10.0)$ & $13(24.5)$ & \\
\hline Bone metastasis & & & & 0.522 \\
\hline No & 85 (69.1) & 50 (71.4) & $35(66.0)$ & \\
\hline Yes & $38(30.9)$ & $20(28.6)$ & $18(34.0)$ & \\
\hline
\end{tabular}




\begin{tabular}{|c|c|c|c|c|}
\hline Clinical characteristics & Overall [n (\%)] & PNI $>46.05[\mathrm{n}(\%)]$ & $\mathrm{PNI} \leq 46.05[\mathrm{n}(\%)]$ & $\begin{array}{l}P \\
\text { value }\end{array}$ \\
\hline PD-L1 expression & & & & 0.962 \\
\hline Positive & $24(19.5)$ & $14(20.0)$ & $10(18.9)$ & \\
\hline Negative & $10(8.1)$ & $6(8.6)$ & $4(7.5)$ & \\
\hline Unknown & $89(72.4)$ & $50(71.4)$ & $39(73.6)$ & \\
\hline Early progression & & & & 0.009 \\
\hline No & $99(80.5)$ & $62(88.6)$ & $37(69.8)$ & \\
\hline Yes & $24(19.5)$ & $8(11.4)$ & $16(30.2)$ & \\
\hline irAEs & & & & 0.694 \\
\hline No & $93(75.6)$ & $52(74.3)$ & $41(77.4)$ & \\
\hline Yes & $30(24.4)$ & $18(25.7)$ & $12(22.6)$ & \\
\hline
\end{tabular}

(A)

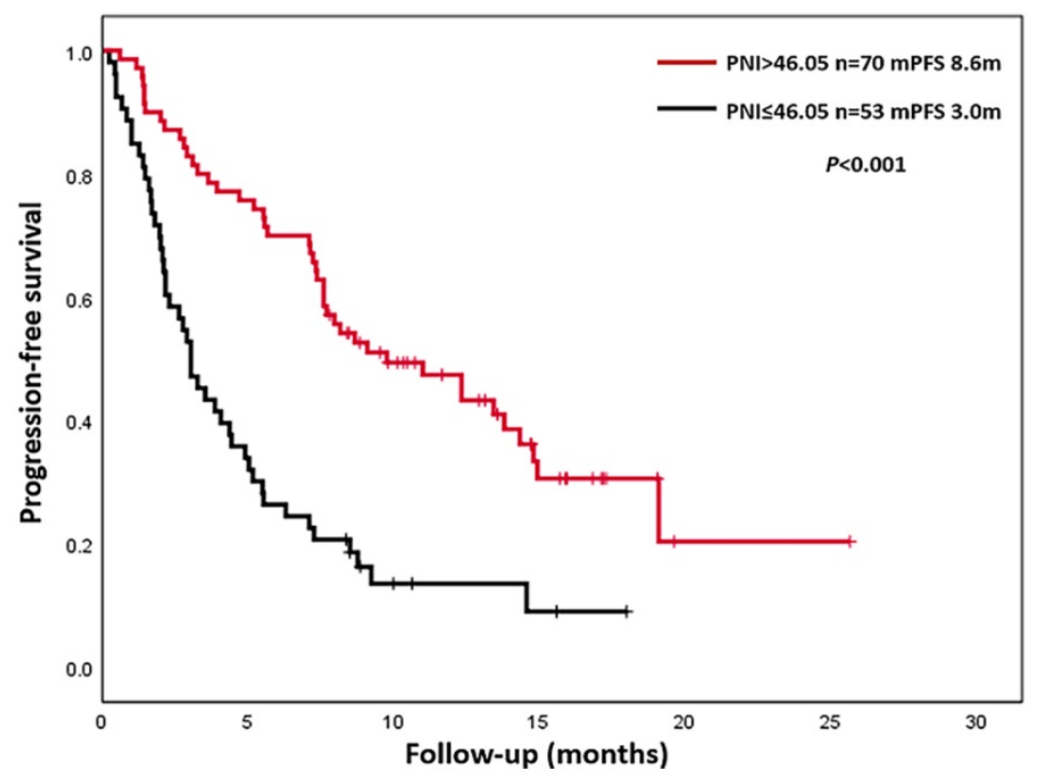

(B)

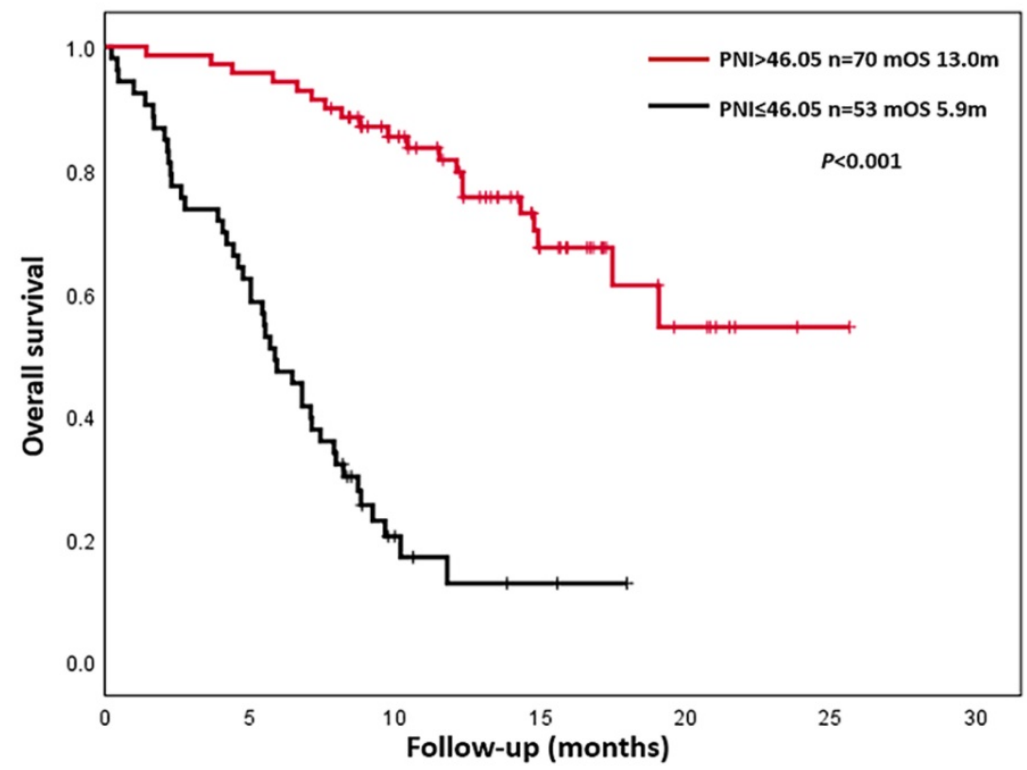

Figure 2. Kaplan-Meier analysis of progression-free survival (PFS) and overall survival (OS). (A) Final descriptive Kaplan-Meier estimates of PFS in all patients as well as stratified by high $(>46.05)$ and low $(\leq 46.05)$ prognostic nutritional index (PNI). (B) Final descriptive Kaplan-Meier estimates of OS in all patients as well as stratified by high (>46.05) and low ( $\leq 46.05)$ PNI.

\section{Survival analyses}

Up to August 30, 2020, a total of 90 patients $(73.2 \%)$ had progressed, and 63 patients $(51.2 \%)$ had died. The causes of death were all related to lung cancer or its complications. The overall PFS was 0.2-25.7 months, and the median PFS (mPFS) was 7.1 months. The overall OS was 0.2-25.7 months, and median OS (mOS) was 12.3 months. K-M survival curves of PFS and OS were drawn according to L-PNI and H-PNI. The results suggested that PFS and OS in H-PNI patients were significantly higher than those in L-PNI patients (mPFS: $8.6 \mathrm{~m}$ vs. $3.0 \mathrm{~m}, P<0.001$; mOS: $13.0 \mathrm{~m}$ vs. $5.9 \mathrm{~m}, P<0.001$ ) (Figure 2). Also, we conducted K-M survival analyses of PFS and OS for LUAD and LUSC patients respectively (Figure S2), and the conclusion was consistent with the overall results.

The univariate analysis suggested that therapy line $\geq 2$, accompanied by driver genomic alterations, bone metastasis, and L-PNI were associated with inferior PFS (Table 2). The multivariate analysis indicated that only PNI (hazard ratio [HR], 2.698, 95\% CI 1.752-4.153; $P<0.001)$ was an independent prognostic factor for PFS (Table 2). Similarly, ECOG PS score 1-2, therapy line $\geq 2$, and L-PNI were related to poorer OS (Table 3). Further multivariate analysis showed that the therapy line (HR, 1.898, 95\% CI, 1.108-3.254; $P=0.011$ ) and PNI (HR, 7.596, 95\% CI, 4.278-13.486; $P<0.001$ ) were independent prognostic factors for OS (Table 3).

\section{Nomogram for overall survival}

Based on the multivariate Cox regression analysis, we identified prognostic predictors (therapy line and PNI) for OS. And we selected them to construct a survival nomogram to predict the 6-, 12- and 18-months OS for advanced NSCLC patients who received PD-1 inhibitors (Figure 3 ). It can conduce to assess the prognosis of patients more intuitively by PNI. We calculated concordance index (C-index), which was 0.777 (95\% CI, 0.611-0.944). The calibration curves indicated the reasonable consistency between the predicted and actual survival probability (Figure 4). 


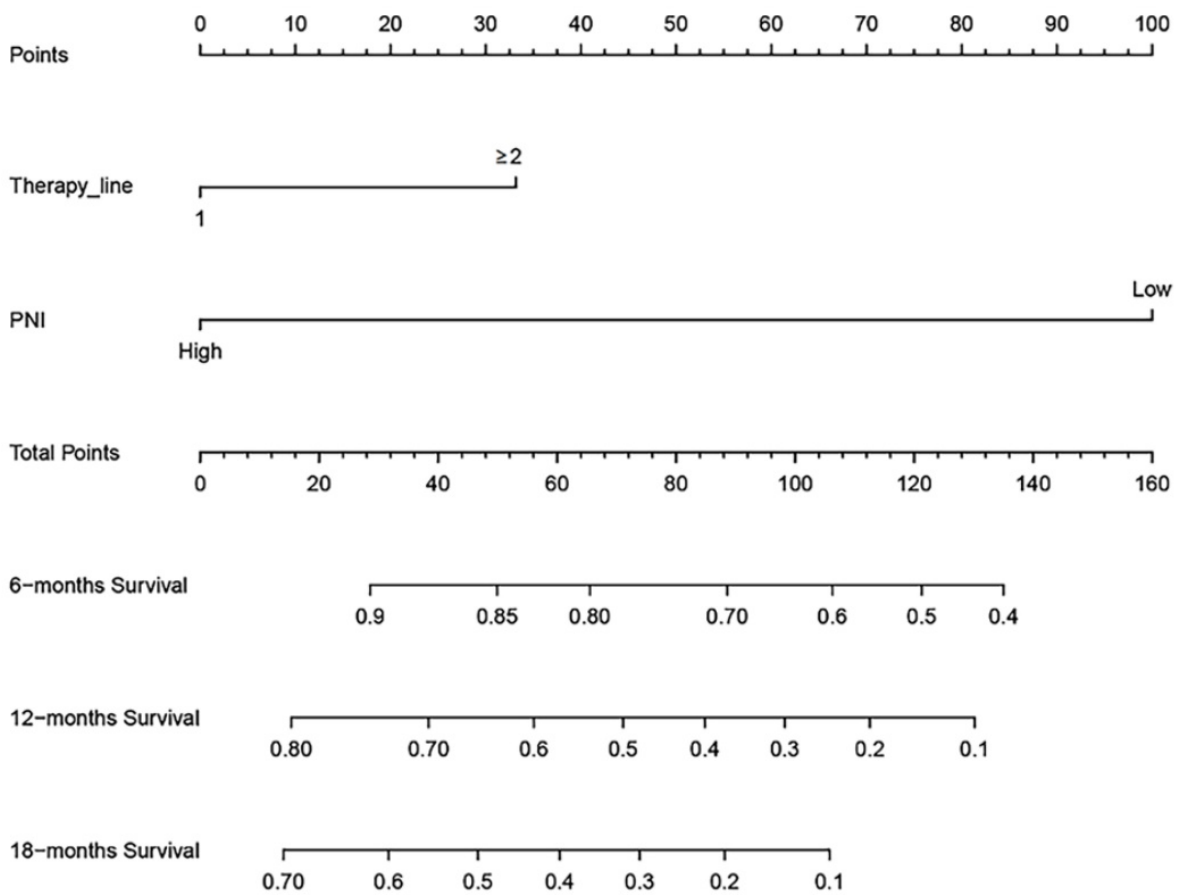

Figure 3. A survival nomogram for 6-, 12- and 18-months overall survival (OS) for non-small cell lung cancer (NSCLC) patients. Nomogram can be interpreted by adding up the points assigned to each variable. The total point projected on the bottom scale represents the probability of 6-, 12- or 18-months OS.

Table 2. Univariate and multivariate analyses of progression-free survival in NSCLC patients treated with PD-1 inhibitors

\begin{tabular}{|c|c|c|c|c|}
\hline \multirow[t]{2}{*}{ Variable } & \multicolumn{2}{|l|}{ Univariate analysis } & \multicolumn{2}{|l|}{ Multivariate analysis } \\
\hline & HR $(95 \% \mathrm{CI})$ & $P$ value & $\mathrm{HR}(95 \% \mathrm{CI})$ & $P$ value \\
\hline Age $(\leq 65$ vs. $>65)$ & $1.107(0.715-1.713)$ & 0.649 & & \\
\hline $\begin{array}{l}\text { Gender } \\
\text { (male vs. female) }\end{array}$ & $1.477(0.911-2.394)$ & 0.113 & & \\
\hline $\begin{array}{l}\text { Smoking status } \\
\text { (never vs. current/ } \\
\text { previous) }\end{array}$ & $0.842(0.552-1.283)$ & 0.423 & & \\
\hline $\begin{array}{l}\text { ECOG PS } \\
(0 \text { vs. } 1-2)\end{array}$ & $1.446(0.940-2.227)$ & 0.094 & & \\
\hline History & & & & \\
\hline LUAD vs. LUSC & $1.144(0.747-1.751)$ & 0.536 & & \\
\hline LUAD vs. other & $1.001(0.243-4.124)$ & 0.999 & & \\
\hline $\begin{array}{l}\text { Stage } \\
\text { (IIIB/IIIC vs. IV) }\end{array}$ & $1.117(0.659-1.896)$ & 0.680 & & \\
\hline $\begin{array}{l}\text { Therapy line } \\
\text { (1 vs. } \geq 2)\end{array}$ & $1.918(1.240-2.966)$ & 0.003 & $1.568(0.979-2.511)$ & 0.061 \\
\hline $\begin{array}{l}\text { Regimen } \\
\text { (monotherapy vs. } \\
\text { combination } \\
\text { therapy) }\end{array}$ & $0.878(0.535-1.439)$ & 0.605 & & \\
\hline $\begin{array}{l}\text { Driver genomic } \\
\text { alterations } \\
\text { (no vs. yes) }\end{array}$ & 1.904 (1.145-3.167) & 0.013 & $1.211(0.683-2.148)$ & 0.512 \\
\hline $\begin{array}{l}\text { Liver metastasis } \\
\text { (no vs. yes) }\end{array}$ & $1.570(0.886-2.783)$ & 0.123 & & \\
\hline $\begin{array}{l}\text { CNS metastasis } \\
\text { (no vs. yes) }\end{array}$ & $1.051(0.603-1.832)$ & 0.859 & & \\
\hline $\begin{array}{l}\text { Bone metastasis } \\
\text { (no vs. yes) }\end{array}$ & $1.577(1.022-2.434)$ & 0.040 & $1.468(0.921-2.340)$ & 0.107 \\
\hline $\begin{array}{l}\text { PD-L1 expression } \\
\text { (no vs. yes) }\end{array}$ & 1.417 (0.514-3.907) & 0.500 & & \\
\hline irAEs (no vs. yes) & $1.089(0.677-1.752)$ & 0.725 & & \\
\hline PNI (high vs. low) & $2.798(1.823-4.292)$ & $<0.001$ & $2.698(1.752-4.153)$ & $<0.001$ \\
\hline
\end{tabular}

Table 3. Univariate and multivariate analyses of overall survival in NSCLC patients treated with PD-1 inhibitors

\begin{tabular}{|c|c|c|c|c|}
\hline \multirow[t]{2}{*}{ Variable } & \multicolumn{2}{|l|}{ Univariate analysis } & \multicolumn{2}{|l|}{ Multivariate analysis } \\
\hline & $\mathrm{HR}(95 \% \mathrm{CI})$ & $P$ value & HR $(95 \% \mathrm{CI})$ & $P$ value \\
\hline Age $(\leq 65$ vs. $>65)$ & $1.306(0.785-2.175)$ & 0.304 & & \\
\hline $\begin{array}{l}\text { Gender } \\
\text { (male vs. female) }\end{array}$ & $0.910(0.492-1.684)$ & 0.765 & & \\
\hline $\begin{array}{l}\text { Smoking status } \\
\text { (never vs. current/ } \\
\text { previous) }\end{array}$ & $1.177(0.700-1.980)$ & 0.538 & & \\
\hline $\begin{array}{l}\text { ECOG PS } \\
(0 \text { vs. } 1-2)\end{array}$ & $1.856(1.119-3.080)$ & 0.017 & $1.536(0.915-2.578)$ & 0.105 \\
\hline \multicolumn{5}{|l|}{ History } \\
\hline LUAD vs. LUSC & $1.268(0.767-2.096)$ & 0.355 & & \\
\hline LUAD vs. other & $0.584(0.080-4.265)$ & 0.596 & & \\
\hline $\begin{array}{l}\text { Stage (IIIB/IIIC } \\
\text { vs. IV) }\end{array}$ & $0.974(0.519-1.828)$ & 0.936 & & \\
\hline $\begin{array}{l}\text { Therapy line } \\
\text { (1 vs. } \geq 2)\end{array}$ & $1.898(1.108-3.254)$ & 0.020 & $2.033(1.178-3.507)$ & 0.011 \\
\hline $\begin{array}{l}\text { Regimen } \\
\text { (monotherapy vs. } \\
\text { combination } \\
\text { therapy) }\end{array}$ & $0.624(0.364-1.071)$ & 0.087 & & \\
\hline $\begin{array}{l}\text { Driver genomic } \\
\text { alterations } \\
\text { (no vs. yes) }\end{array}$ & $1.294(0.689-2.431)$ & 0.423 & & \\
\hline $\begin{array}{l}\text { Liver metastasis } \\
\text { (no vs. yes) }\end{array}$ & $1.292(0.637-2.620)$ & 0.477 & & \\
\hline $\begin{array}{l}\text { CNS metastasis } \\
\text { (no vs. yes) }\end{array}$ & $1.568(0.834-2.945)$ & 0.162 & & \\
\hline $\begin{array}{l}\text { Bone metastasis } \\
\text { (no vs. yes) }\end{array}$ & $1.566(0.941-2.606)$ & 0.085 & & \\
\hline $\begin{array}{l}\text { PD-L1 expression } \\
\text { (no vs. yes) }\end{array}$ & $1.835(0.514-6.548)$ & 0.350 & & \\
\hline irAEs (no vs. yes) & $0.734(0.399-1.352)$ & 0.322 & & \\
\hline PNI (high vs. low) & $7.596(4.278-13.486)$ & $<0.001$ & $7.222(4.081-12.781)$ & $<0.001$ \\
\hline \multicolumn{5}{|c|}{$\begin{array}{l}\text { Abbreviation: NSCLC, non-small cell lung cancer; PD-1, programmed death 1; HR, } \\
\text { hazard ratio; CI, confidence interval; ECOG PS, Eastern Cooperative Oncology } \\
\text { Group performance status; LUAD, lung adenocarcinoma; LUSC, lung squamous } \\
\text { cell carcinoma; CNS, central nervous system; PD-L1, programmed death-ligand 1; } \\
\text { irAEs, immune-related adverse events; PNI, prognostic nutritional index. }\end{array}$} \\
\hline
\end{tabular}


(A)

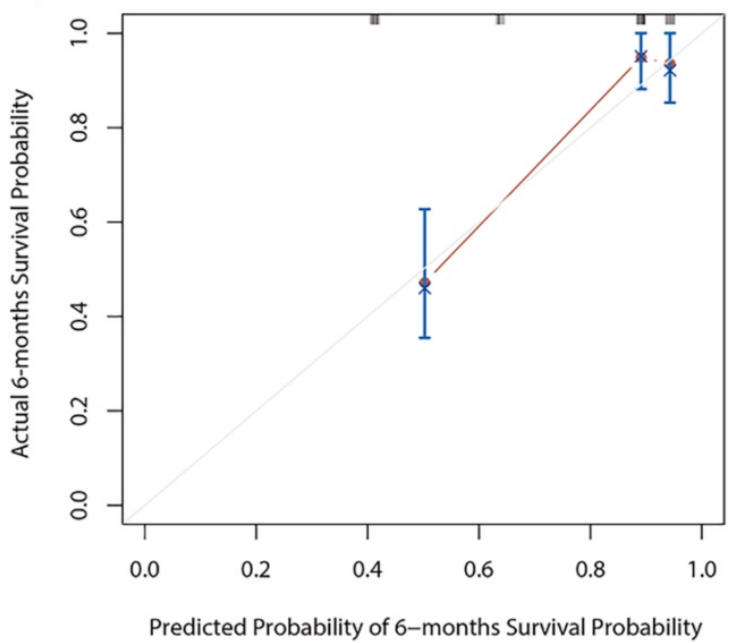

(B)

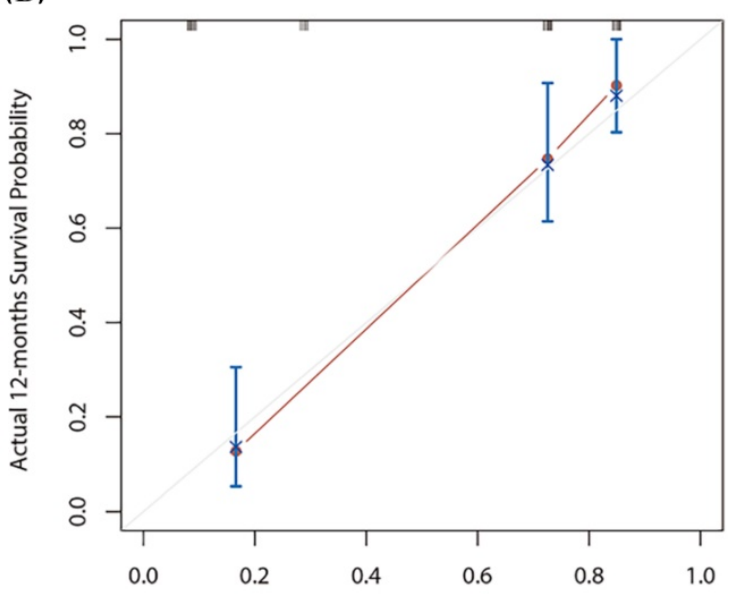

Predicted Probability of 12-months Survival Probability

(C)

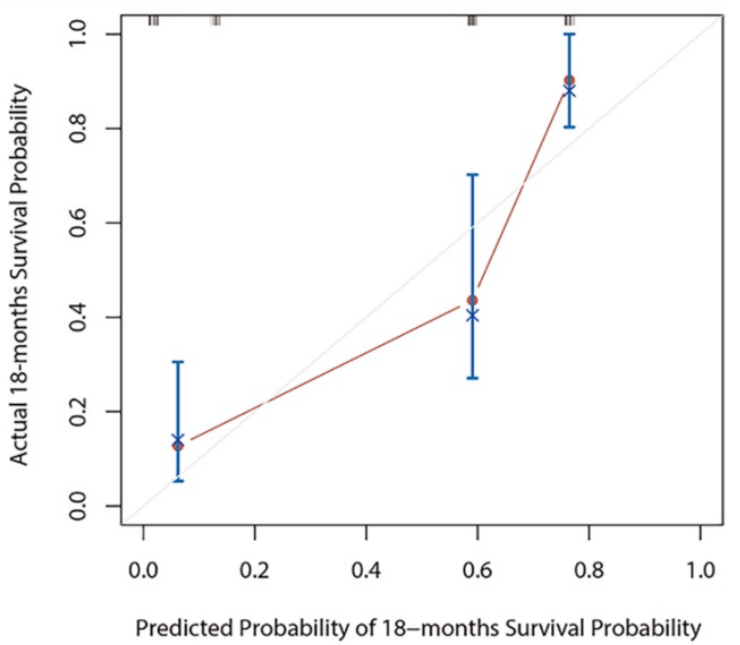

Figure 4. The calibration curves for 6-, 12- or 18-months overall survival (OS) nomogram $(A-C)$. The $X$ axis is nomogram predicted survival probability and $Y$ axis is actual survival probability.

\section{Discussion}

Immunotherapy has been approved more indications in patients with advanced NSCLC. From nivolumab monotherapy to pembrolizumab and atezolizumab single or combination therapy, immunotherapy has established its position in the treatment of advanced NSCLC. Several clinical trials have reported durable responses and long-term survival benefits of immunotherapy. However, how to identify the patients most likely to benefit from treatment remains a challenge at present. Therefore, exploring some predictive biomarkers can contribute to selecting the population with better response to immunotherapy, thereby maximizing the benefits of patients.

Although the assessment of tumor and tumor microenvironment is essential to determine biomarkers for immunotherapy, host-related factors, particularly in nutrition and immune status, cannot be ignored. Serum albumin level is a common indicator to evaluate the nutritional status of patients. Low albumin level reflects poor nutritional status, weakens many defense mechanisms of the body, such as cellular and humoral immunity and phagocyte function, and is closely related to the poor prognosis of cancer patients $[17,18]$. For patients with advanced cancer, pretreatment lymphocytopenia is a poor prognostic factor, which may be associated with pre-existing immunosuppressive conditions. It has been reported that the absolute lymphocyte count level can be used as an alternative indicator to represent the host immune level and predict the overall treatment outcomes in cancer patients [19]. PNI integrates serum albumin and lymphocyte levels, which can reflect the host immune-nutritional status of patients. This indicates that it has potential predictive value for advanced NSCLC patients treated with PD-1 inhibitors.

Our study evaluated the early progression and prognosis of advanced NSCLC patients who received PD-1 inhibitors in the clinical practice of our hospital. The results elucidated that pretreatment PNI is a reliable and independent predictor of early progression, PFS, and OS. In the multivariate analysis, the therapy line was also associated with early progression and OS. The predictive role of PNI has been extensively researched in multiple malignancies [20, 21, 22]. Preoperative PNI was relevant to postoperative lung complications and long-term prognosis, and can predict the postoperative outcomes of lung cancer in elderly patients [23, 24]. Recently, a meta-analysis including 15 articles discussed the prognostic role of PNI in patients with NSCLC [25]. The result indicated that low PNI was a reliable indicator of poor OS $(P<0.001)$, and it was also a useful indicator of disease-free survival, recurrence-free survival, and PFS [25]. Moreover, previously published studies also suggested that low 
PNI was a poor prognostic indicator for NSCLC patients who received EGFR-tyrosine kinase inhibitors and was associated with shorter OS [26, 27]. Shoji et al. retrospectively analyzed the pretreatment PNI level of 102 NSCLC patients who received ICIs treatment [28]. The results suggested that PNI was significantly relevant to the objective response rate (ORR), disease control rate, and PFS in those patients. It also showed a trend as an independent prognostic indicator for OS, but did not reach a statistically significant level (RR, 1.606, $P=0.0761$ ) [28]. In our study, PNI was a significant independent prognostic indicator for OS (HR, 7.222, 95\% CI, 4.081-12.781, $P<$ 0.001), and was superior to PFS (HR, 2.698, 95\%CI, $1.752-4.153, P<0.001)$. Overall, $\mathrm{PNI}$ is expected to be a simple and novel predictive biomarker for advanced NSCLC patients treated with ICIs, and may help identify patients who will benefit from ICIs treatment.

Our study suggested that the risk of early progression in L-PNI patients was 3.709 times higher than that in H-PNI patients. This may be correlated with the poor nutritional status of patients, the low host immune function, and the inability of PD-1 inhibitors to arouse the anti-tumor effect of the body. Due to the increased risk of early progression in L-PNI patients and the complexity of cancer immunotherapy, the response rate of monotherapy still has considerable space for improvement $[5,6,29]$. Therefore, further combination chemotherapy may more benefit in L-PNI patients.

Bone metastasis leads to cancer pain, negative effect on physical conditions and deterioration in quality of life, which also affects the survival outcomes of patients. Recently, Landi et al. evaluated the impact of bone metastases on NSCLC patients treated with nivolumab [30]. The results showed that, regardless of the pathological types, ECOG PS status, liver or brain metastases and bone palliative radiotherapy, patients with positive bone metastases had poor ORR, PFS, and OS [30]. This suggested that organ-specific metastasis may be a prognostic factor for individual immunotherapy. Our result showed that bone metastasis was a prognostic factor for PFS in the univariate analysis, but did not achieve the same result in multivariate analysis. Besides, ECOG PS status also affected the prognosis of patients treated with immunotherapy [31, 32]. Our study concluded that the immunotherapy line was also statistically significant for early progression and survival outcomes of advanced NSCLC patients. It suggested that immunotherapy in initial lines may make advanced NSCLC patients obtain better survival benefits.

Nomogram is a reliable and practical tool for predicting individual survival probability, and its performance is evaluated by consistency index and calibration curves. In this study, we constructed a survival nomogram for 6-, 12- and 18-months OS for advanced NSCLC patients, including the two risk factors of PNI and therapy line. The result suggested that our nomogram of 6- and 12-months had good predictive accuracy.

Despite the advantages of our study, there are also several inevitable limitations that need to be concerned. First of all, this study is a retrospective single-center study with small sample size. Although it can provide certain clinical insights for patient selection, more prospective clinical research are need to verify our conclusions. Besides, insufficient follow-up duration, inconsistent PNI cut-off values, individual differences in nutritional status, comorbidity, and other factors may affect the accuracy of the conclusion. Finally, due to the limited data available, we only evaluated the pretreatment PNI level.

Nevertheless, our research has certain implications for predicting patient clinical outcomes. It can be used as a consideration factor for doctors to choose different patients for ICIs treatment rationally, and can conduce to identify patients with early progression and poor prognosis. The practicability and convenience in the clinic also promote this biomarker to serve clinical decision-making better. Research on the dynamic changes of PNI time-series can also be considered in the future. Besides, exploratory studies related to biomarkers are critical to developing effective approaches to identify and verify the response of ICIs. The prediction model established by PNI combined with different types of biomarkers (such as PD-L1, TMB, and TILs) may be more suitable for clinical application.

\section{Conclusion}

In summary, the present study suggested that PNI is a favorable predictive indicator to evaluate the risk of early progression and survival outcomes in patients with advanced NSCLC during PD-1 inhibitors treatment. Still, prospective studies with further expansion of the sample size are needed to verify and support our conclusions.

\section{Abbreviations}

PNI: prognostic nutritional index; NSCLC: non-small cell lung cancer; PD-1: programmed death 1; CI: confidence interval; HR: hazard ratio; LC: lung cancer; OS: overall survival; ICIs: immune checkpoint inhibitors; PD-L1: programmed death ligand-1; TMB: tumor mutation burden; TILs: tumor-infiltrating lymphocytes; MMR: mismatch repair; ECOG PS: Eastern Cooperative Oncology Group Performance 
Status; PFS: progression-free survival; ROC: receiver operating characteristic; K-M: Kaplan-Meier; LUAD: lung adenocarcinoma; LUSC: lung squamous cell carcinoma; EGFR: epidermal growth factor receptor; ROS1: ROS1 proto-oncogene receptor tyrosine kinase; CNS: central nervous system; irAEs: immune-related adverse events; C-index: concordance index; ORR: objective response rate.

\section{Supplementary Material}

Supplementary figures and tables. http://www.jcancer.org/v12p2960s1.pdf

\section{Acknowledgements}

This article was supported by the International Science and Technology Cooperation Program of Shaanxi Province (2019KW-074).

\section{Ethics committee approval and patient consent}

The study was approved by the Ethics Committee of the First Affiliated Hospital of Xi'an Jiaotong University (XJTU1AF2020LSK-141).

\section{Funding}

This article was supported by the International Science and Technology Cooperation Program of Shaanxi Province (2019KW-074).

\section{Competing Interests}

The authors have declared that no competing interest exists.

\section{References}

1. Siegel RL, Miller KD, Jemal A. Cancer statistics, 2020. CA Cancer J Clin. 2020; 70: 7-30

2. Molina JR, Yang P, Cassivi SD, et al. Non-small cell lung cancer: epidemiology, risk factors, treatment, and survivorship. Mayo Clin Proc. 2008; 83: 584-94.

3. Reck M, Rodríguez-Abreu D, Robinson AG, et al. Pembrolizumab versus Chemotherapy for PD-L1-Positive Non-Small-Cell Lung Cancer. N Engl J Med. 2016; 375: 1823-33.

4. Mok TSK, Wu YL, Kudaba I, et al. Pembrolizumab versus chemotherapy for previously untreated, PD-L1-expressing, locally advanced or metastatic non-small-cell lung cancer (KEYNOTE-042): a randomised, open-label, controlled, phase 3 trial. Lancet. 2019; 393: 1819-30.

5. Gandhi L, Rodríguez-Abreu D, Gadgeel S, et al. Pembrolizumab plus Chemotherapy in Metastatic Non-Small-Cell Lung Cancer. N Engl J Med. 2018; 378: 2078-92.

6. Gadgeel SM, Garassino MC, Esteban E, et al. KEYNOTE-189: Updated OS and progression after the next line of therapy (PFS2) with pembrolizumab (pembro) plus chemo with pemetrexed and platinum vs placebo plus chemo for metastatic nonsquamous NSCLC. J Clin Oncol. 2019; 37.

7. Paz-Ares L, Luft A, Vicente D, et al. Pembrolizumab plus Chemotherapy for Squamous Non-Small-Cell Lung Cancer. N Engl J Med. 2018; 379: 2040-51.

8. Fehrenbacher L, Spira A, Ballinger M, et al. Atezolizumab versus docetaxel for patients with previously treated non-small-cell lung cancer (POPLAR): a multicentre, open-label, phase 2 randomised controlled trial. Lancet. 2016; 387 : $1837-46$

9. Rittmeyer A, Barlesi F, Waterkamp D, et al. Atezolizumab versus docetaxel in patients with previously treated non-small-cell lung cancer (OAK): a phase 3 , open-label, multicentre randomised controlled trial. Lancet. 2017; 389: 255-65.

10. Hellmann MD, Nathanson T, Rizvi H, et al. Genomic Features of Response to Combination Immunotherapy in Patients with Advanced Non-Small-Cell Lung Cancer. Cancer Cell. 2018; 33: 843-52.e4.
11. Yarchoan M, Albacker LA, Hopkins AC, et al. PD-L1 expression and tumor mutational burden are independent biomarkers in most cancers. JCI Insight. 2019; 4: e126908.

12. Candido J, Hagemann T. Cancer-related inflammation. J Clin Immunol. 2013; 33 (Suppl 1): S79-S84

13. Mayne ST, Playdon MC, Rock CL. Diet, nutrition, and cancer: past, present and future. Nat Rev Clin Oncol. 2016; 13: 504-15.

14. Onodera T, Goseki N, Kosaki G. Prognostic nutritional index in gastrointestinal surgery of malnourished cancer patients. Nihon Geka Gakkai Zasshi. 1984; 85: 1001-5.

15. Bozkaya $\mathrm{Y}, \mathrm{Köstek} \mathrm{O}$, Sakin A, et al. Is the prognostic nutritional index a prognostic and predictive factor in metastatic non-small cell lung cancer patients treated with first-line chemotherapy? Support Care Cancer. 2020; 28: 2273-82.

16. Ozdemir Y, Topkan E, Mertsoylu H, et al. Low Prognostic Nutritional Index Predicts Poor Clinical Outcomes in Patients with Stage IIIB Non-small-cell Lung Carcinoma Undergoing Chemoradiotherapy. Cancer Manag Res. 2020; 12: 1959-67.

17. Wolf G, Schroeder R, Ziyadeh FN, et al. Albumin up-regulates the type II transforming growth factor-beta receptor in cultured proximal tubular cells. Kidney Int. 2004; 66: 1849-58.

18. Liu J, Dai Y, Zhou F, et al. The prognostic role of preoperative serum albumin/ globulin ratio in patients with bladder urothelial carcinoma undergoing radical cystectomy. Urol Oncol. 2016; 34: 484.e1-.e8.

19. Ray-Coquard I, Cropet C, Van Glabbeke M, et al. Lymphopenia as a prognostic factor for overall survival in advanced carcinomas, sarcomas, and lymphomas. Cancer Res. 2009; 69: 5383-91.

20. Go SI, Park S, Kang MH, et al. Clinical impact of prognostic nutritional index in diffuse large B cell lymphoma. Ann Hematol. 2019; 98: 401-11.

21. Huang $\mathrm{X}, \mathrm{Hu} \mathrm{H}$, Zhang $\mathrm{W}$, et al. Prognostic value of prognostic nutritional index and systemic immune-inflammation index in patients with osteosarcoma. J Cell Physiol. 2019; 234: 18408-14

22. Okadome K, Baba Y, Yagi T, et al. Prognostic Nutritional Index, Tumorinfiltrating Lymphocytes, and Prognosis in Patients with Esophageal Cancer. Ann Surg. 2020; 271: 693-700.

23. Park S, Ahn HJ, Yang $M$, et al. The prognostic nutritional index and postoperative complications after curative lung cancer resection: A retrospective cohort study. J Thorac Cardiovasc Surg. 2020; 160: 276-85.e1.

24. Watanabe I, Kanauchi N, Watanabe H. Preoperative prognostic nutritional index as a predictor of outcomes in elderly patients after surgery for lung cancer. Jpn J Clin Oncol. 2018; 48: 382-7.

25. $\mathrm{Hu} \mathrm{Y}$, Shen J, Liu R, et al. Prognostic value of pretreatment prognostic nutritional index in non-small cell lung cancer: A systematic review and meta-analysis. Int J Biol Markers. 2018; 33: 372-8.

26. Park $\mathrm{S}$, Park $\mathrm{S}$, Lee $\mathrm{SH}$, et al. Nutritional status in the era of target therapy: poor nutrition is a prognostic factor in non-small cell lung cancer with activating epidermal growth factor receptor mutations. Korean J Intern Med. 2016; 31: 1140-9.

27. Sheng J, Yang YP, Ma YX, et al. Low Prognostic Nutritional Index Correlates with Worse Survival in Patients with Advanced NSCLC following EGFR-TKIs. PLoS One. 2016; 11: e0147226.

28. Shoji F, Takeoka H, Kozuma Y, et al. Pretreatment prognostic nutritional index as a novel biomarker in non-small cell lung cancer patients treated with immune checkpoint inhibitors. Lung Cancer. 2019; 136: 45-51.

29. Langer CJ, Gadgeel SM, Borghaei $\mathrm{H}$, et al Carboplatin and pemetrexed with or without pembrolizumab for advanced, non-squamous non-small-cell lung cancer: a randomised, phase 2 cohort of the open-label KEYNOTE-021 study. Lancet Oncol. 2016; 17: 1497-508.

30. Landi L, D'Incà F, Gelibter $\mathrm{A}$, et al. Bone metastases and immunotherapy in patients with advanced non-small-cell lung cancer. J Immunother Cancer. 2019; 7: 316.

31. Garde-Noguera J, Martin-Martorell P, De Julián M, et al. Predictive and prognostic clinical and pathological factors of nivolumab efficacy in non-small-cell lung cancer patients. Clin Transl Oncol. 2018; 20: 1072-9.

32 Dall'Olio FG, Magoio I, Massucci M, et al. ECOG performance status $\geq 2$ as a prognostic factor in patients with advanced non small cell lung cancer treated with immune checkpoint inhibitors-A systematic review and meta-analysis of real world data. Lung Cancer. 2020; 145: 95-104. 